\section{Broadband printed circularly-polarised aperture antenna array for millimetre-wave gigabit applications}

\section{K.-F. Hung and Y.-C. Lin}

A simple and successful design of a printed aperture antenna array is presented in view of high-speed millimetre-wave wireless access applications. The antenna element design is based on a broadband travelling-wave aperture antenna for circularly-polarised radiation. For the antenna array design, the sequential rotation scheme is employed for improving the co-polarised gain and axial ratio. The presented antenna array is designed in the $40-48 \mathrm{GHz}$ band and implemented with a 5mil-thick RT/Duroid-5880 PCB. Promising performances are achieved, including a return loss $(<10 \mathrm{~dB})$ bandwidth of $32 \%$, an axial ratio $(<3 \mathrm{~dB})$ bandwidth of $25 \%$, and a co-polarised gain (flatness $<3 \mathrm{~dB}$ ) bandwidth of $32 \%$ with a maximum gain of $11 \mathrm{dBic}$

Introduction: In recent years, there have been a variety of wireless communication applications aiming to utilise millimetre-wave (MMW) frequencies for broadband radio links. In particular, the increasing demands for high-speed (with data rates $>1 \mathrm{Gbit} / \mathrm{s}$ ) transmission in the short-range MMW wireless personal area network (WPAN) have grown rapidly in the past few years $[1,2]$. Associated with these applications is the need for a directive, broadband, and low-cost antenna that can be easily integrated with the MMW circuitry of a transceiver module/packaging [3]. To fulfil all these requirements, the MMW antenna design has become a key component in system implementation.

Several printed MMW antenna designs have been studied and reported in the literature recently. In [4], a linear array of aperture coupled stacked patch antennas with reflector patches is proposed for improving the front-to-back ratio and gain level for $K a$-band LMDS wireless applications. Another approach [5] is using a broadband folded dipole array to directly integrate with MMW transceiver ICs for $60 \mathrm{GHz}$ WPAN applications. However, in these studies, the antennas are designed for linear polarisation, which necessitates rotating the transmitting and receiving antenna properly for polarisation matching, particularly in the case of the line-of-sight (LOS) radio link. Therefore, a circularly-polarised antenna is desirable in view of MMW wireless access applications.

In this Letter we present the design and implementation of a broadband circularly-polarised aperture antenna array for MMW wireless applications. The presented antenna array has following features: 1. the low-cost planar PCB design suitable for mass production, 2. the simple microstrip-fed design for easy interfaces, 3. the cavity-backed design for the unidirectional patterns, and 4 . the broadband capability for high-speed MMW wireless applications.

Antenna structure and design concept: Fig. 1 shows the configuration of the presented antenna with the co-ordinate systems and design parameters. A circular aperture of diameter $D_{a}$ is etched out from the ground plane of a PCB. Above the aperture is a tapered strip fed by a microstrip transmission line printed on the opposite side of the PCB. The tapered strip is designed with a curved shape formed by two equiangular curves, $C_{1}$ and $C_{2}$, written as the following equations in polar co-ordinates

$$
\begin{aligned}
& C_{1}: \rho=\left(D_{a} / 2\right) e^{-a \phi} \\
& C_{2}: \rho=\left(D_{a} / 2-t\right) e^{-b \phi}
\end{aligned}
$$

where $a$ and $b$ are shrinking coefficients of curve $C_{1}$ and $C_{2}$, respectively, and $t$ is the initial width of the tapered strip at the input point. The metal strip is exponentially tapered and extended towards the centre of the aperture with a terminated angle, denoted by $\phi_{\mathrm{e}}$. As described in the prior research [6], the aperture antenna generates right-handed circularly-polarised waves with bidirectional patterns. In this Letter, the antenna is further developed with a backed cavity for generating unidirectional patterns. The cavity is specified by a diameter $D_{c}$ and height $H_{c}$. The simulation of the design work is conducted with a full-wave electromagnetic simulator [7]. The proposed antenna design is illustrated with the right-handed circular polarisation (RCP) in the 40 $48 \mathrm{GHz}$ band. The prototype is implemented with an RT/Duroid-5880 PCB with the dielectric constant of 2.2 , loss tangent of 0.0009 , and thickness of $5 \mathrm{mil}$.

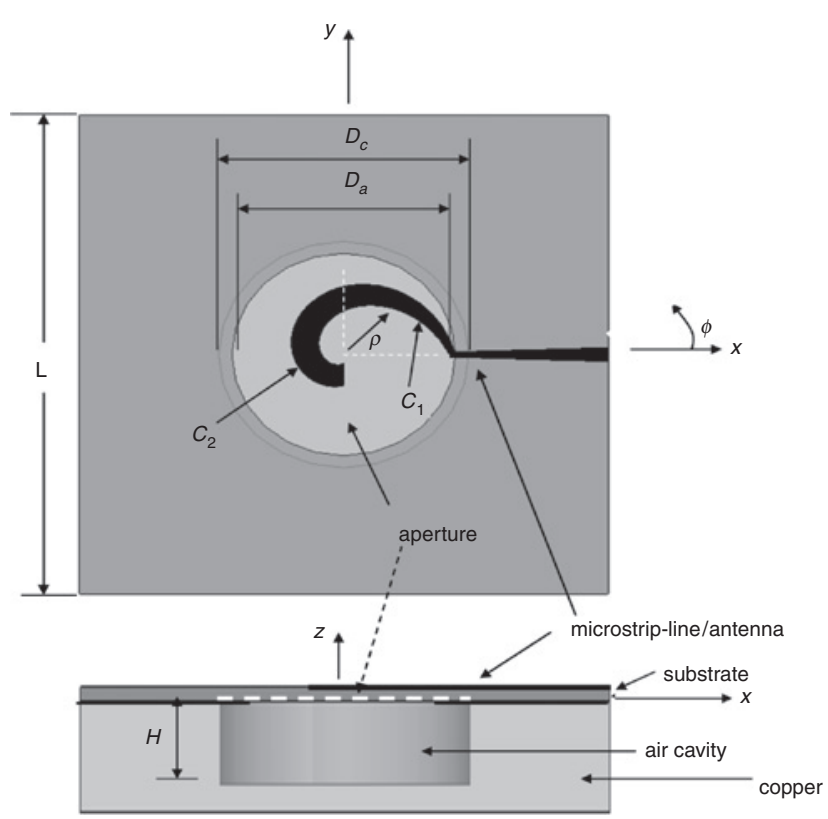

Fig. 1 Antenna configuration and co-ordinate systems

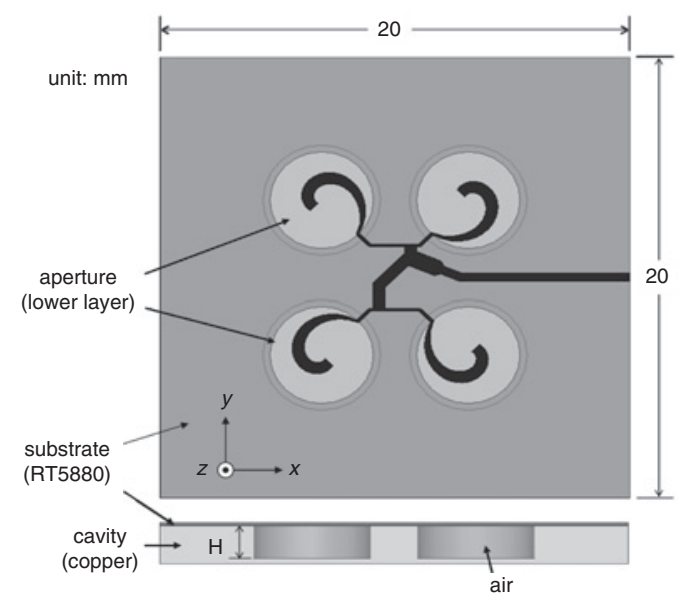

Fig. 2 Top and side view of presented $2 \times 2$ antenna array with backed cavities

Antenna array and experimental results: Fig. 2 shows the configuration of the experimental $2 \times 2$ array using the aforementioned aperture antennas for radiating elements. The antenna elements are spaced at 6 and $7 \mathrm{~mm}$ along $x$ - and $y$-axis, respectively. They are also sequentially rotated $90^{\circ}$ counter-clockwise (for RCP sense). A corporate feeding network is devised to excite each rotated element with the phase sequentially delayed by $90^{\circ}$. This sequential rotation scheme had been proven an effective technique for improving the axial ratio of a circularlypolarised antenna array [8]. The corporate feeding networks are designed with sequential phased and impedance transformation. From the extensive simulation, the optimised dimensions of the aperture antenna are obtained with $a=0.5, b=0.3, D_{a}=4.4 \mathrm{~mm}, D_{c}=$ $5 \mathrm{~mm}, \phi_{\mathrm{e}}=1.5 \pi$, and $H=2 \mathrm{~mm}$. Once the aperture antennas and feeding networks are printed on the PCB, it is then placed on top of a metal fixture with embedded machined cavities. Finally, a $50 \Omega$ $\mathrm{V}$-connector $(1.85 \mathrm{~mm})$ with a glass bead is connected to the input port for measurements. The measured and simulated return loss of the developed $2 \times 2$ antenna array is shown in Fig. 3. The measured $10 \mathrm{~dB}$ return loss bandwidth is about $32 \%$ (38 to $52 \mathrm{GHz}$ ). Note that the measurements agree with the simulation results in that the $1.85 \mathrm{~mm} \mathrm{~V}$-connector is included. Fig. 4 shows the measured and simulated radiation patterns of the developed $2 \times 2$ array in two elevation cuts ( $x z$ - and $y z$-plane) at $44 \mathrm{GHz}$. The co-polarised (RCP) gain at zenith is about $11 \mathrm{dBic}$ with a front-to-back ratio about $25 \mathrm{~dB}$. Fig. 5 shows the measured and simulated frequency response of the RCP gain and axial ratio observed at zenith (z-axis). The $3 \mathrm{~dB}$ axial ratio bandwidth is found to be better than $25 \%$ (out of scope). The measured 
$\mathrm{RCP}$ gain at zenith has a half-power $(3 \mathrm{~dB})$ gain bandwidth better than $32 \%$ with maximum gain of $11 \mathrm{dBic}$.

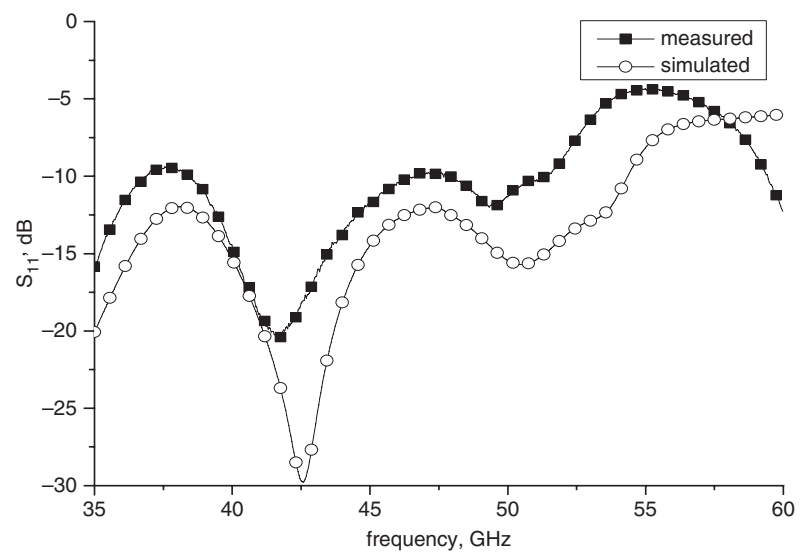

Fig. 3 Measured and simulated return loss of developed $2 \times 2$ array
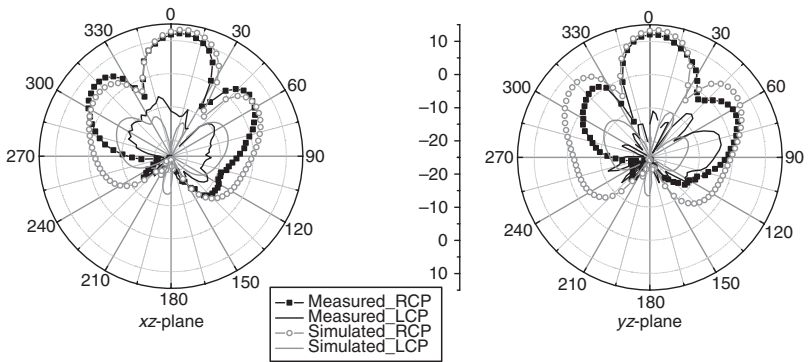

Fig. 4 Measured and simulated radiation patterns in two elevation cuts of presented $2 \times 2$ array

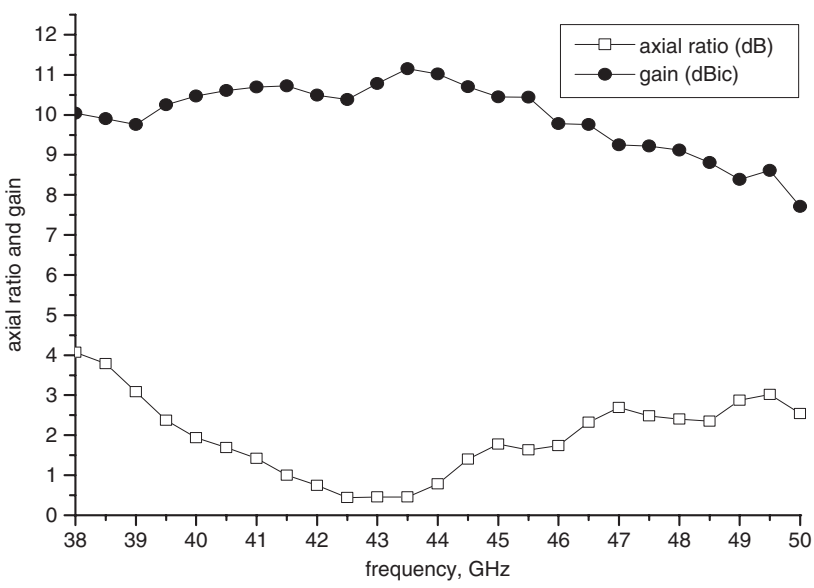

Fig. 5 Measured frequency response of RCP gain and axial ratio at zenith of developed $2 \times 2$ array
Conclusion: In this Letter, a broadband microstrip-fed circularlypolarised aperture antenna array is presented. The proposed antenna array is characterised of wide bandwidth, high gain, and low manufacturing cost. The broadband characteristics of the antenna array are achieved in terms of the return loss bandwidth, the axial ratio bandwidth, and the gain bandwidth. All these good performance make it a good candidate for high data-rate MMW wireless applications.

Acknowledgments: This work was supported in part by the National Science Council (NSC), Taiwan, under contract numbers NSC 952219-E-002-007 and NSC 96-2752-E-002-002-PAE and in part by Excellent Research Projects of National Taiwan University under grant number 97R0062-03.

(C) The Institution of Engineering and Technology 2008 3 September 2008

Electronics Letters online no: 20082551

doi: 10.1049/el:20082551

K.-F. Hung (Graduate Institute of Communication Engineering, National Taiwan University, Taipei, Taiwan)

Y.-C. Lin (Department of Electrical Engineering, National Taiwan University, Taipei, Taiwan)

E-mail: yclin@cc.ee.ntu.edu.tw

\section{References}

1 Tsukamoto, K., et al.: 'Development of gigabit millimeter-wave broadband wireless access system-system overview'. Proc. AsiaPacific Microwave Conf., Seoul, Korea, 2003, pp. 957-960

2 Ogawa, H.: 'Millimeter-wave wireless personal area network systems'. Proc. Radio Frequency Integrated Circuits Symp., San Francisco, CA, USA, June 2006, pp. 11-13

3 Tsai, J.-H., Wang, T.-P., Lin, K.-Y., Huang, T.-W., Lin, Y.-C., Lu, H.-C., and Wang, H.: '40-48 GHz sub-harmonic transceiver for high data-rate communication system applications'. Millimeter Waves GSMM 2008 Global Symp., Nanjing, China, April 2008, pp. 234-237

4 Waterhouse, R.B., Novak, D., Nirmalathas, A., and Lim, C.: 'Broadband printed sectorized coverage antennas for millimeter-wave wireless applications', IEEE Trans. Antennas Propag., 2002, 50, (1), pp. 12-16

5 Zwick, T., Liu, D., and Gaucher, B.P.: 'Broadband planar superstrate antenna for integrated millimeter-wave transceivers', IEEE Trans. Antennas Propag., 2006, 54, (10), pp. 2790-2796

6 Hung, K.-F., and Lin, Y.-C.: 'Simulation of single-arm fractional spiral antennas for millimeter wave applications'. Antennas and Propagation Society Int. Symp., Albuquerque, NM, USA, July 2006, pp. 3697-3700 7 Ansoft HFSS V10

8 Kraft, U.R.: 'Main-beam polarization properties of four-element sequential-rotation arrays with arbitrary radiators', IEEE Trans. Antennas Propag., 1996, 44, (4), pp. 515-522 